\title{
Individuals expend more effort to compete against robots than humans after observing competitive human-robot interactions
}

\author{
Rosanne H. Timmerman*11 Te-Yi Hsieh*1, Anna Henschel\# ${ }^{1}$, Ruud Hortensius\# ${ }^{1,2}$ and Emily S. Cross\# ${ }^{1,3}$ \\ *co-first authors \#co-corresponding authors
}

\begin{abstract}
In everyday life, we often observe and learn from interactions between other individuals - so-called third-party encounters. As robots are poised to become an increasingly familiar presence in our daily lives, third-party encounters between other people and robots might offer a valuable approach to influence people's behaviours and attitudes towards robots. Here, we conducted an online experiment where participants $(n=48)$ watched videos of human-robot dyads interacting in a cooperative or competitive manner. Following this observation, we measured participants' behaviour and attitudes towards the human and robotic agents. First, participants played a game with the agents to measure whether their behaviour was affected by their observed encounters. Second, participants' attitudes toward the agents were measured before and after the game. We found that the third-party encounters influenced behaviour during the game but not attitudes towards the observed agents. Participants showed more effort towards robots than towards humans, especially when the human and robot agents were framed as competitive in the observation phase. Our study suggests that people's behaviours towards robots can be shaped by the mere observation of third-party encounters between robots and other people.
\end{abstract}

Keywords: human-robot interaction, third-party encounters, social robotics, artificial agents, social cognition

\section{INTRODUCTION}

We frequently observe interactions among others - socalled third-party encounters. These encounters influence people's attitudes towards the observed individuals and, if positive, can serve as an easily implemented and unthreatening tool to reduce prejudice towards minority groups, unfamiliar individuals and outgroups [1]-[9]. Effects of third-party encounters can be equal to or even surpass those brought about by direct contact [10]. These effects of vicarious contact persist over time and can generalize beyond the observed agents [11], [12]. For example, it was found that children prefer the friends of people receiving positive non-verbal signals over friends of people receiving negative signals [12].

*Research supported by funding from the European Research Council (ERC) under the European Union's Horizon 2020 research and innovation programme (Grant agreement number 677270 to E.S.C.), the Leverhulme Trust (PLP-2018-152 to E.S.C), and the BIAL Foundation (to R.H.).

1 Institute of Neuroscience and Psychology, School of Psychology, University of Glasgow, Scotland, UK

2 Department of Psychology, Utrecht University, Heidelberglaan 1, 3584 CS, Utrecht, The Netherlands

3 Department of Cognitive Science, Macquarie University, Sydney, Australia
Third-party encounters may hold great practical potential in human-robot interaction (HRI). While social robots are becoming more prevalent in daily life [13], negative attitudes towards robots still persist [14]. In order to reduce people's negative attitudes towards robots, third-party encounters have been proposed as a possible solution by researchers in the field [14]-[17]. For instance, Fraune and colleagues [16] showed that watching positive HRIs increased people's willingness to interact with robots.

So far, most research has focused on the impact of thirdparty encounters on observers' attitudes and only limited empirical evidence has suggested that these encounters will induce behaviour change in the observer, specifically towards the observed agents [7]. Skinner and colleagues [12] found that observing an interaction between people can change some daily behaviours unspecific to the observed people. Someone receiving positive non-verbal signals by the other person in the observed encounter were more likely to influence children in using the same label for an object than a person receiving negative signals [12]. Yet, more detailed research on behavioural change is essential to lay the foundations for robust HRI. For example, people who have negative attitudes towards robots have been also shown to behave more negatively towards robots during real-life interactions [17].

The aims of the current exploratory study were to replicate findings that attitudes towards robots can be changed by observing HRIs [1]-[9] and to investigate potential behaviour change in observers [7]. Specifically, we set out to examine whether observing videos of cooperative versus competitive HRIs influence how people perceive similar agents and behave towards them. To answer this, we conducted an online experiment where participants observed human-robot dyads acting cooperatively or competitively and assessed participants' attitude changes and motivation to engage with each observed agent in a game. Participants were led to believe that they were playing against an algorithm based on pre-recorded behaviour of the agents. Based on the previous findings, we evaluated the following general expectations:

1. People show a difference in motivation when engaging in the game with agents framed as cooperative versus competitive.

2. Participants will show differential preferences for cooperative compared to competitive agents. This is based on findings from Correia and colleagues [15], who showed that robots cooperating with the team were rated more positively than a robot following its own goal, regardless of the game result. 
We further explored the extent to which opponent type (human versus robot) influenced behaviour and attitude.

\section{METHODS}

\section{A. Data Accessibility Statement}

Materials, data and code for all experiments are available on the OSF https://osf.io/uvy3b/. We report all measures in the study, all manipulations, any data exclusions and the sample size determination rule.

\section{B. Participants}

Forty-eight participants, of which 16 were female (M age $=26.2, \mathrm{SD}=6.8 ;$ sex of one participant remained unspecified) were recruited via Prolific (www.prolific.co). As a rule of thumb, we determined the sample size by multiplying the number of participants recruited in a comparable study by two. Specifically, we used the study by Walbrin and colleagues, Experiment 2 as reference $(n=23)$ [18]. The main experiment was described as watching videos of human and robotic agents followed by playing a game with these agents. To increase the believability of the online experimental setting, participants were told that they would play against algorithms based on these previously observed agents. Participants received $£ 2.52$ for their participation in the study (equivalent to $£ 6.73 /$ hour). To increase motivation, participants were told that the top $10 \%$ had a chance of receiving a bonus payment of $£ 5$. Inclusion criteria were an approval rate of $100 \%$ on the Prolific website and no participation in the validation and pilot studies prior to the main experiment (see below). Participants were naive to the goal of the study, most of them (87.5\%) were unfamiliar with the robot used in the study and had little or no experience in interacting with robots in daily life (median on a scale from 1 (never) to 7 (daily) was 2 with an interquartile range of 1). The experiments were designed in PsychoPy3 and later uploaded to Pavlovia (https://pavlovia.org/; [19]) an online experiment platform. The whole experiment took approximately 20 minutes. Participants provided informed consent before the start of the experiment. The study procedure was approved by the Research Ethics Committee of the College of Science and Engineering at the University of Glasgow (protocol number: 300180301).

\section{Experimental design}

We used a two-by-two (agent type: human or robot; agent intention: cooperative or competitive) within-subjects factorial design to examine the impact of agent type, agent intention, and the interaction between these two factors on participants' attitudes and behaviours towards the observed agents.

\section{Stimuli}

Participants watched 2 short videos (10 seconds) of a human and robot playing a bar game together, which served as a framing story to the main task. In these videos (Fig. 1A and 1B), a bar was located in the middle of the screen between two opposite goals, one in the upper and one in the lower part of the screen. The agents moved their arms either up or down, giving the impression that they controlled the movement of the bar. In the cooperative condition, the human and robot appeared to work together to reach the same goal by moving their arm in the same direction simultaneously. In the competitive condition, both agents tried to reach opposite goals by moving their arms in opposite directions (Fig. 1B). The purpose of the videos was to frame each agent as either competitive or cooperative. Later in the experiment, participants engaged in a bar game similar to this one with one of the agents (either the cooperative robot, cooperative human, competitive robot or competitive human). The bar game looked almost identical to the one in the framing videos, but the participants could now actively move the bar upwards by pressing the space bar. Again, there were two goals, the upper one belonging to the participant (marked by a square called "YOU") and the lower one belonging to the agent. The videos were edited in DaVinci Resolve v15.3.1 1 [20]. The agents were filmed in front of a green screen, which was later removed and replaced by the bar game. Three validation studies (first validation: $n=20$, second validation: $n=12$, third validation: $n=40$ ) were conducted in order to improve and select the most salient stimuli for the main experiment. The third validation study (containing the videos for the main experiment) showed that agents were consistently rated as either cooperative or competitive, on a slider from ' 1 ' as 'competitive' to ' 7 ' as 'cooperative' (cooperative human: $M$ $=5.60, S D=1.74$, cooperative robot: $M=5.97, S D=1.48$, competitive human: $M=2.35, S D=1.85$, competitive robot: $M=2.17, S D=1.75$ ) (Fig. 2D). To avoid possible gender bias effects, we generated two different orders: order A in which the female human agent was the cooperative agent and the male was the competitive agent, and order B where the male human agent was the cooperative agent and the female the competitive agent. For all analyses, no differences were found between the two orders.

\section{E. Measures}

To operationalize people's motivation to play against agents, we measured the number of space bar presses during the bar game. Participants played three games with each agent, and each game was at a different difficulty level (easy, medium or hard). Difficulty levels were manipulated to measure subtle differences in motivation and to increase believability of the bar game. The different difficulty levels were defined by: 1) the number of times the participant had to press the space bar before it could be moved upwards (from easy to hard respectively: 2, 4, 6), and 2) how many times the bar would move downwards towards the goal of the opponent agent $(150,100,50)$. Thus, in the easy condition participants had to press the space bar the least number of times to move it upwards and the bar would move downwards the least. The resulting 12 games were played in a randomized order. A game ended either when ten seconds had passed or when one of the players reached one of the goals. After the game round ended, the score was presented and participants could see how many times they pressed the space bar, if they reached the goal, and if they received a penalty, as well as their total scores. Participants would get a penalty ( -5 points) if they did not let go of the space bar (instead of pressing "space" repeatedly). Participants could get one penalty per game, thus the maximum amount of 
penalties per participant was 48. The given amount of penalties were low $(M=1.79, S D=3.75)$. We interpreted a higher number of space presses as increased competitiveness and effort put into the game.

Measures on participants' attitudes towards agents involved three parts: First, participants' preference towards agents was measured before (pre-preference) and after (postpreference) the game by preferential ranking from "most preferable" to "least preferable" to play a game with. The pre- and post-measurements were implemented because we anticipated that an effect of the third-party encounters would be stronger in pre-preference (i.e., right after watching the framing videos) than in post-preference ratings (i.e., after playing the games where all agents played the role of a game opponent). Second, the perceived cooperativeness and socialness towards agents was determined by slider ratings from competitive to cooperative, and from individual to social. Last, participants' decisions of whether an agent was cooperative or competitive was acquired by using a twoalternative forced choice task.

\section{F. Experimental procedure}

The main experiment consisted of four parts. First, participants observed two framing videos to learn the roles of each agent (cooperative or competitive agents). While watching the videos, they were instructed to pay attention specifically to one of the agents. One of the videos showed agents cooperating with each other, while the other showed two agents competing against each other. Each framing video was repeated twice for each agent. Prior to the video demonstration, instruction videos were provided to
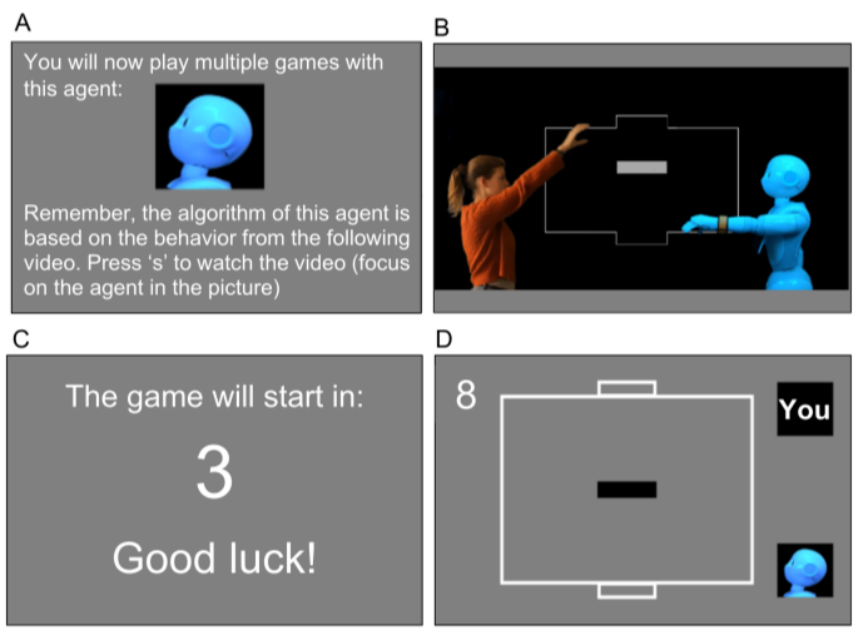

E

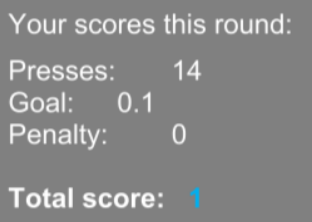

Figure 1. Bar game design. (A) The bar game began with an agent's picture signifying participants' next opponent. (B) This was followed by a framing video. (C) Countdown to prepare the participant for the game. (D) The bar game lay-out. (E) Presentation of the scores were presented after each game. communicate the workings of the bar game, i.e., when an agent moved its arm, the bar would move accordingly. To check whether participants paid attention to the videos, they were asked whether the specified agent had reached the goal. Following the third-party encounters, participants ranked the agents from most to least preferable to play a game with. Next, participants played a bar game with each agent in a semi-randomized order. Before the game, participants read a cover story that suggested they were actually playing against an algorithm based on the observed agents' game behaviour. Participants were told that the behaviours were modelled and created by using a deep neural network. The story was accompanied by an image of a schematic explanation of a deep neural network. The bar game (Fig. 1D) began with an agent's picture signifying this round's opponent (Fig. 1A). To remind participants of the intention of the agent, the framing video was shown again (Fig. 1B). There was a countdown announcing the start of each game (Fig. 1C). After each game participant were shown their scores (Fig. $1 \mathrm{E})$.

In the final part, each agent was rated on their socialness and cooperativeness levels. Ratings were placed at the end so that participants could form their own opinions throughout the experiment. Finally, we asked participants to describe the algorithm in their own words to check whether they believed the cover story. The free text responses showed that the words most used to describe the agents were 'computer' $(n=51)$, 'man' $(n=22)$, 'woman' $(n=21)$ and 'robot' $(n=17)$. It is not surprising that the agents are most often described as computers since a computer is often the layman's interpretation of an algorithm. For instance, many people have experiences with bots, which function as software applications (e.g. chatbots). The fact that the robotic and human agents were described as 'robot', 'man' and 'woman', indicates participants believed they were playing against these agents (i.e., their pre-recorded behaviour).

\section{G. Data processing and analysis}

All data analyses were carried out in R v4.0.1 [22]. For the behavioural data of the numbers of space bar presses, we ran a linear mixed effects model with the lme4 package (v1.1.23) [23] to examine if participants' game behaviours were influenced by agent type (human or robot) and agent intention (cooperative or competitive) while controlling the random individual differences (Prolific_id), trial differences (trial_number), and the random effects by game difficulty levels (difficulty_level). The model building started from the maximal random effect components [24], and we reduced the complexity, resulting in the following formula: numbers of presses agent_type*agent_intention + (1+agent_type |Prolific_id $)+(1 \mid$ trial_number $)+(1 \mid$ difficulty_level $)$.

The analyses regarding participants' attitudes toward each agent was done in three parts. First, participants' ordinal ranking of the most preferable agent to the least preferable was analysed via a mixed effects ordinal regression model with the ordinal package (v2019.12.10) [25]. We tested the fixed effects of agent type (human or robot), agent intention (cooperative or competitive), and ranking timing (pre-game 
or post-game) on people's ordinal preferences, while controlling the random effects of participants (Prolific_id) and the random order of the four agents introduced to each participant (present_order). The final model that converged was ranking agent_type*agent_intention*rank_time + $(1+$ agent_type*agent_intention|Prolific_id $)+\quad(1+$ agent_type*agent_intention|present_order).

Second, participants' slider ratings of the agents' cooperativeness and socialness were analysed via two linear mixed effects models respectively. For the cooperativeness model, agent type (human or robot) and agent intention (cooperative or competitive) were included as fixed effects, and the final random effect structure which led to model convergence involved: by-subject random intercepts, and random slopes for the effects of agent type and agent intention on subjects: cooperativeness_rating agent_type* agent_intention $+(1+$ agent_type + agent_intention (Prolific_id). The socialness model was similarly designed, except that it included an additional random factor of order (order A or B): socialness_rating agent_type* agent_intention $+(1+$ agent_type + agent_intention |Prolific_id $)+(1$ order $)$.

Third, we analysed participants' binomial forced choices on whether an agent was cooperative or competitive via a mixed effects logistic regression model with the "glmer" function in

lme4 package (v1.1.23). We examined the fixed effects of agent type (human or robot) and agent intention (cooperative or competitive) on participants choices, while controlling by-

subject random intercepts and random slopes for agent type on subjects: forced_choice agent_type + agent_intention + (1+agent_type|Prolific_id). All linear data were centred by the grand mean before model building. When conducting pairwise post-hoc tests, p-values were adjusted using Tukey's method. All analysis code can be accessed on our dedicated OSF page for this project: https://osf.io/uvy3b/.

\section{RESULTS}

\section{A. Behavioural results (bar game)}

The result of the mixed effects model showed a significant main effect of agent type $(\beta=3.08,95 \%$ CI [0.78, 5.38], $p=.009)$, and a significant interaction between agent type and agent intention $(\beta=-3.03,95 \% C I[-5.42,-0.65], p$ $=.013$ ) on the numbers of times participants pressed the space bar. No main effect of agent intention was observed $(\beta$ $=0.59,95 \%$ CI $[-1.09,2.28], p=.491)$. In general, participants pressed the space bar more often when playing against robots $(M=27.87, S D=21.86)$ than against humans ( $M=26.30, S D=23.03$ ) (Fig. 2A). Pairwise post-hoc tests on the interaction between agent type and intention were carried out with the emmeans package (v1.4.7) [26]. When playing against the competitive robot $(M=29.09, S D=22.25)$, participants pressed the space bar more often than when playing against the cooperative robot $(M=26.65, S D=$ 21.40), $t(2193.0)=2.84, p=.024$ (Fig. 2B). However, no clear difference emerged when comparing the competitive robot and the competitive human $(M=26.00, S D=23.08)$, $t(87.6)=-2.63, p=.049$. Likewise, there was no significant difference found in the comparisons between competitive human and cooperative human $(M=26.60, S D=22.99)$, $t(2193.0)=-0.69, p=.902$, competitive human and cooperative robot, $t(87.6)=-0.55, p=.947$, competitive robot and cooperative human, $t(87.6)=2.12, p=.154$, or cooperative human and cooperative robot, $t(87.6)=-0.04, p=$ 1.000 .

\section{B. Attitude results}

\section{Preferential ranking of agents}

In the result of our mixed effects ordinal regression model, agent type (odds ratio $=1.34,95 \% C I$ [0.37, 4.90], $p$ $=.658)$, agent intention (odds ratio $=1.19,95 \%$ CI $[0.34$, 4.09], $\mathrm{p}=.787$ ), or ranking timing (odd ratio $=1.16,95 \% C I$ $[0.50,2.67], p=.724)$ did not influence participants' preferential ranking towards the four agents.

\section{Cooperativeness slider rating}

Participants' cooperativeness ratings were significantly influenced by the interaction between agent type and agent intention $(\beta=-0.28,95 \% C I[-0.52,-0.04], p=.02)$, but not by the main effect of agent type $(\beta=-0.06,95 \% C I[-0.58$, $0.41], p=.804)$ or agent intention $(\beta=0.19,95 \% C I[-0.32$, $0.71], p=.462)$. The cooperative human agent was rated as the most cooperative $(M=3.81, S D=1.71)$ by participants, whereas the cooperative robot was the most competitive $(M=$ $3.47, S D=1.42$ ) among the four agents (competitive human: $M=3.62, S D=1.63$; competitive robot: $M=3.56, S D=$ 1.59). However, follow-up post hoc tests did not reveal any significant differences in the following pairs: competitive human vs. cooperative human $(t(52.5)=-0.74, p=.883)$; competitive human vs. competitive robot $(t(53.8)=0.25, p=$ $.995)$; competitive human vs. cooperative robot $(t(47.0)=$ $0.44, p=.972)$; cooperative human vs. competitive robot $(t(47.0)=0.72, p=.888)$; cooperative human vs. cooperative robot $(t(53.8)=1.43, p=.486)$; competitive robot vs. cooperative robot $(t(52.5)=0.34, p=.986)$ (Fig. 2C). This is in contrast with the ratings in the validation study, where we observed a very clear distinction in cooperativeness slider ratings between the agents that were framed as cooperative and competitive in the videos (Fig. 2D).

\section{Socialness slider rating}

Participants' socialness ratings were significantly impacted by agent type $(\beta=-0.55,95 \% C I[-0.96,-0.15], p=$ $.008)$ but not by agent intention $(\beta=0.12,95 \%$ CI $[-0.30$, $0.54], p=.577)$ nor the interaction between agent type and intention $(\beta=-0.20,95 \%$ CI [-0.42, 0.02], $p=.079)$. Participants rated humans $(M=3.79, S D=1.50)$ as more social than robots $(M=3.14, S D=1.33)$.

\section{Cooperativeness forced choice}



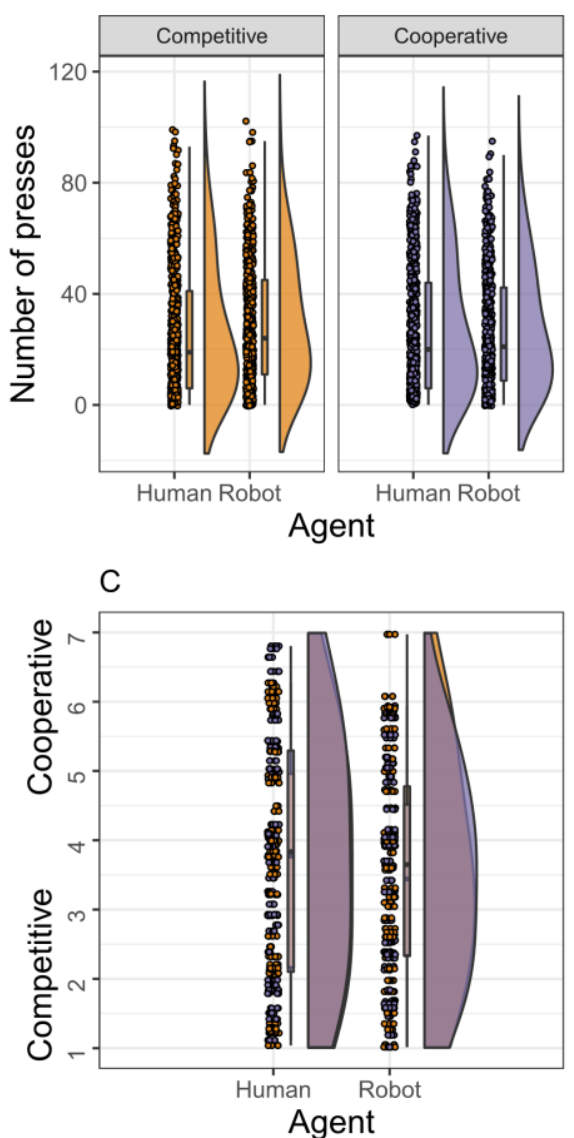

B
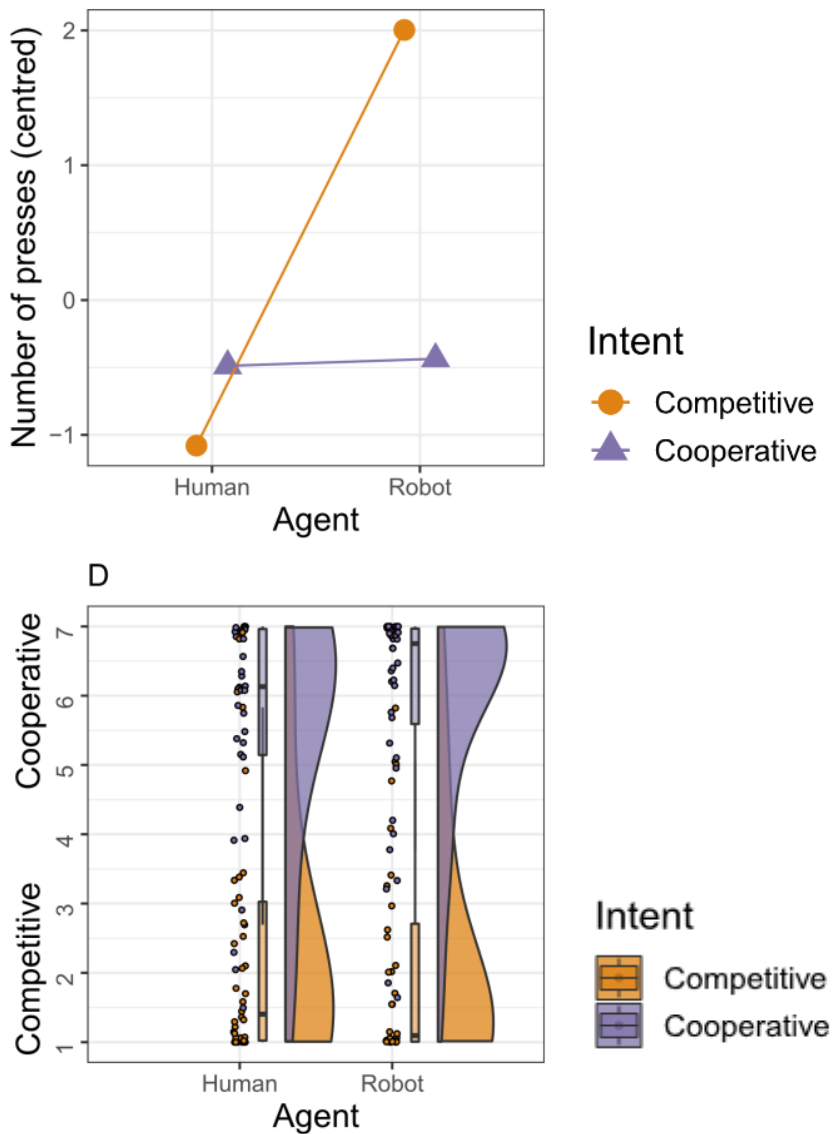

Figure 2. (A) Number of presses in bar game per agent. (B) Interaction between agent and intent on centred number of presses. (C) Cooperativeness ratings per agent in main experiment. (D) Cooperativeness ratings per agent in validation experiment.

Neither agent type (odds ratio $=0.46,95 \%$ CI $[0.11$, $1.93], p=.288$ ) nor agent intention (odds ratio $=0.74,95 \%$ $C I[0.50,1.08], p=.118$ ) was found to influence participants' forced choices of whether an agent was cooperative or competitive. This is surprising, given that in the validation study there was a clear strong effect of intent on the forced choices. Agents were consistently labelled as either cooperative (cooperative human: $n=32$, cooperative robot: $n$ $=33$ ) or competitive (competitive human: $n=34$, competitive robot: $n=34)$.

\section{DISCUSSION}

We investigated the impact of human-robot encounters on people's attitudes and behaviours in the context of online games. Participants observed human-robot dyads interacting either cooperatively or competitively and were then led to believe they were playing a competitive game against algorithms informed by these agents' behaviours (while in reality playing against the computer).

Third-party encounters influenced participant's game competitiveness, but not attitude towards the observed agents. The main finding in our study was that participants showed higher game competitiveness (i.e., pressed the space bar more frequently) toward robotic agents than human agents, especially when the agents were framed as competitive in the observation phase. However, the findings on the attitude change towards robots were inconsistent. Our results suggest that people's perceived cooperativeness of the agents was influenced by the interaction between agent type and agent intention, and that people perceived human agents as more social than robotic agents. Below we discuss these findings in detail.

Participants' increased game competitiveness towards robot compared to human opponents is in line with previous research, in which participants behaved more competitively toward a robot than a human in economic games [27]. Our study further showed that such discriminatingly competitive behaviours toward robots could be diminished by observing cooperative human-robot encounters before actual HRI. After participants had observed the human and robotic agents cooperating in the short video clips, they responded to cooperative robots and cooperative humans equally in the competitive online game. Notably, the effect of humanrobot encounters we found on game behaviours existed regardless of the agents' actual behaviours when people directly interacted with them. Participants in the present study showed the highest game competitiveness towards the robot framed as competitive in the observation phase, even though all agents' game behaviours (behavioural competitiveness) remained consistent according to the preprogrammed difficulty levels. These finding highlights the effects of third-party encounters on observers' game behaviours, and relates to previous studies documenting the 
persistent impact of first impressions of robots on people's behaviours during HRIs [28], [29].

Regarding the impact of human-robot encounters on participants' attitudes towards the agents, our results were inconsistent. Firstly, encounters of cooperative and competitive human-robot dyads had no effect on participants' agent preference rankings, neither in the prepreference nor in the post-preference tasks. This might suggest that the third-party encounters of cooperative and competitive HRI were irrelevant and thus did not influence people's preferences towards the agents in our experiment. A study by Huisman et al. [30] showed that the perceived politeness of virtual robots is not affected by cooperativeness or competitiveness of an agent during a game. However, other research has found that perceived warmth, competence, and personality of a robot are more crucial factors in our preferences towards robots [31], [32]. Future studies could consider manipulating these factors in human-robot encounters to investigate the subsequent impact on people's preferences towards the observed agents.

The impact of cooperative and competitive human-robot encounters on people's perceived cooperativeness towards the agents was not robust in our study. Our post hoc analyses did not reveal any difference in either pair of the four agents, albeit the significant interaction between agent type (human/robot) and agent intention (cooperative/competitive) emerged for participants' cooperativeness slider rating towards the agents. Similarly, participants' forced choices regarding the cooperative and competitive nature of an agent were not influenced by agent intention framing or agent type. These results suggest that the agent intention manipulation was perhaps not strong enough to shape participants' perceived cooperativeness of the agents, which contradicted the results of our validation studies where competitive and cooperative framing was accurately differentiated by participants' cooperativeness rating. Another possible explanation for the ineffective agent intention manipulation was the competitive nature of the game. In the bar game, the participants and the agents had opposite goals to achieve and therefore all the agents might be perceived as competitive by the participants. This competitive game experience may obscure the manipulation of agent intention in the prior observation phase. Previous studies have pointed out that the perceived competitiveness in environments or agents can shape people's attitudes. For examples, Mutlu et al. (2006) found that people had more positive attitudes towards the ASIMO robot in a cooperative game context than in a competitive game context [33]. Even when researchers did not intend to frame the robot as competitive, participants can be sensitive to robot's noncooperative decisions and responded to these reciprocally [34]. Therefore, future research on this topic may choose to make more judicious decisions when designing a HRI context and manipulating an agent's intention, to ensure an agent's attribute in third-party encounters is not in contrast with how the agents behave in the actual HRI.

Finally, our study revealed a significant effect of agent type (human/robot) on participants' socialness ratings towards the observed agents. Human agents were rated as more social than robotic agents. This is not surprising since we have extensive social experience with human interaction partners, whereas robots are only emerging in social contexts. However, as robots are becoming more prevalent, it could be possible that, by manipulation of robots' characteristics, robots' perceived socialness can be amplified [35]. It would be valuable for future research to explore whether third-party encounters of robots with different characteristics lead to varying degrees of perceived socialness, as well as to further identify the key factors shaping the attribution of socialness to robots. All in all, the current study provides fundamental evidence concerning the influence of observed human-robot encounters on people's behaviours towards the observed agents. Specifically, in our online game environment, participants showed more game competitiveness in competing against the robot previously framed as competitive than the robot framed as cooperative. However, this work warrants further research on the generalisability of people's behaviours during competitive games to behaviours in real-world HRI. Future research should also explore the impact of human-robot encounters in embodied HRI instead of online interactions. Although online studies can provide insightful evidence [36]-[38], physical embodiment has been regarded as an important factor shaping our perception and behaviours towards robots [35], [39]-[41]. For example, people would experience more enjoyment and engagement in embodied HRI than in virtual HRI [39]. Future research could extend the current exploration on human-robot encounters to other more realistic contexts, or to other aspects of social behaviours, and further substantiate the link between people's attitudes and behaviours. By doing so, researchers might be able to unveil the potential utility of third-party encounters to promote the social quality of reallife HRI, and to better embed social robots into human society.

\section{REFERENCES}

[1] J. D. Johnson and L. Ashburn-Nardo, 'Testing the "Black Code": Does Having White Close Friends Elicit Identity Denial and Decreased Empathy From Black In-Group Members?', Soc. Psychol. Personal. Sci., vol. 5, no. 3, pp. 369-376, Apr. 2014, doi: $10.1177 / 1948550613499938$.

[2] A. Mazziotta, A. Mummendey, and S. C. Wright, 'Vicarious intergroup contact effects: Applying social-cognitive theory to intergroup contact research', Group Process. Intergroup Relat., vol. 14, no. 2, pp. 255-274, Mar. 2011, doi: 10.1177/1368430210390533.

[3] S. Quadflieg and I. S. Penton-Voak, 'The Emerging Science of People-Watching: Forming Impressions From Third-Party Encounters', Curr. Dir. Psychol. Sci., vol. 26, no. 4, pp. 383-389, Aug. 2017, doi: 10.1177/0963721417694353.

[4] S. Quadflieg and K. Westmoreland, 'Making Sense of Other People's Encounters: Towards an Integrative Model of Relational Impression Formation', J. Nonverbal Behav., vol. 43, no. 2, pp. 233-256, Jun. 2019, doi: 10.1007/s10919-019-00295-1.

[5] J. R. Shapiro, M. Baldwin, A. M. Williams, and S. Trawalter, 'The company you keep: Fear of rejection in intergroup interaction', $J$. Exp. Soc. Psychol., vol. 47, no. 1, pp. 221-227, Jan. 2011, doi: 10.1016/j.jesp.2010.10.006.

[6] L. Vezzali, G. A. Di Bernardo, S. Stathi, E. P. Visintin, and M. Hewstone, 'Using intercultural videos of direct contact to implement vicarious contact: A school-based intervention that improves intergroup attitudes', Group Process. Intergroup Relat., vol. 22, no. 7, pp. 1059-1076, Oct. 2019, doi: 10.1177/1368430218809885. 
[7] K. West and R. Turner, 'Using extended contact to improve physiological responses and behavior toward people with schizophrenia', J. Exp. Soc. Psychol., vol. 50, pp. 57-64, Jan. 2014, doi: 10.1016/j.jesp.2013.06.009

[8] G. Willard, K.-J. Isaac, and D. R. Carney, 'Some evidence for the nonverbal contagion of racial bias', Organ. Behav. Hum. Decis. Process., vol. 128, pp. 96-107, May 2015, doi:

10.1016/j.obhdp.2015.04.002.

[9] P. Winkler et al., 'Short video interventions to reduce mental health stigma: a multi-centre randomised controlled trial in nursing high schools', Soc. Psychiatry Psychiatr. Epidemiol., vol. 52, no. 12, pp. 1549-1557, Dec. 2017, doi: 10.1007/s00127-017-1449-y.

[10] A. Eller, D. Abrams, and A. Gomez, 'When the direct route is blocked: The extended contact pathway to improving intergroup relations', Int. J. Intercult. Relat., vol. 36, no. 5, pp. 637-646, Sep. 2012, doi: 10.1016/j.ijintrel.2012.03.005.

[11] G. Lemmer and U. Wagner, 'Can we really reduce ethnic prejudice outside the lab? A meta-analysis of direct and indirect contact interventions', Eur. J. Soc. Psychol., vol. 45, no. 2, pp. 152-168, 2015, doi: 10.1002/ejsp.2079.

[12] A. L. Skinner, A. N. Meltzoff, and K. R. Olson, "Catching” Social Bias: Exposure to Biased Nonverbal Signals Creates Social Biases in Preschool Children', Psychol. Sci., vol. 28, no. 2, pp. 216-224, Feb. 2017, doi: 10.1177/0956797616678930.

[13] E. Broadbent, 'Interactions With Robots: The Truths We Reveal About Ourselves', Annu. Rev. Psychol., vol. 68, no. 1, pp. 627-652, Jan. 2017, doi: 10.1146/annurev-psych-010416-043958.

[14] R. Wullenkord and F. Eyssel, 'Improving attitudes towards social robots using imagined contact', 23rd IEEE Int. Symp. Robot Hum. Interact. Commun., 2014, doi: 10.1109/ROMAN.2014.6926300.

[15] F. Correia et al., 'Exploring Prosociality in Human-Robot Teams', in 2019 14th ACM/IEEE International Conference on Human-Robot Interaction (HRI), Daegu, Korea (South), Mar. 2019, pp. 143-151, doi: 10.1109/HRI.2019.8673299.

[16] M. R. Fraune, B. C. Oisted, C. E. Sembrowski, K. A. Gates, M. M. Krupp, and S. Šabanović, 'Effects of robot-human versus robot-robot behavior and entitativity on anthropomorphism and willingness to interact', Comput. Hum. Behav., vol. 105, p. 106220, Apr. 2020, doi: 10.1016/j.chb.2019.106220.

[17] T. Nomura, T. Kanda, T. Suzuki, and K. Kato, 'Prediction of Human Behavior in Human--Robot Interaction Using Psychological Scales for Anxiety and Negative Attitudes Toward Robots', IEEE Trans. Robot., vol. 24, no. 2, pp. 442-451, Apr. 2008, doi: 10.1109/TRO.2007.914004

[18] J. Walbrin, P. Downing, and K. Koldewyn, 'Neural responses to visually observed social interactions', Neuropsychologia, vol. 112, pp. 31-39, Apr. 2018, doi: 10.1016/j.neuropsychologia.2018.02.023.

[19] J. Peirce et al., 'PsychoPy2: Experiments in behavior made easy', Behav. Res. Methods, vol. 51, no. 1, pp. 195-203, Feb. 2019, doi: 10.3758/s13428-018-01193-y.

[20] DaVinci Resolve Engineering Team, 'DaVinci Resolve 15.3 Reference Manual [Internet] (15.3) [Computer software]. Blackmagic Design.' 2019

[21] J. Pfau, J. D. Smeddinck, I. Bikas, and R. Malaka, 'Bot or not? User Perceptions of Player Substitution with Deep Player Behavior Models', in Proceedings of the 2020 CHI Conference on Human Factors in Computing Systems, Honolulu HI USA, Apr. 2020, pp. 110, doi: $10.1145 / 3313831.3376223$.

[22] R Core Team, ' $\mathrm{R}$ : a language and environment for statistical computing [Internet]'. Foundation for Statistical Computing., 2020.

[23] D. Bates, M. Mächler, B. Bolker, and S. Walker, 'Fitting Linear Mixed-Effects Models using lme4', ArXiv14065823 Stat, Jun. 2014, Accessed: Jan. 25, 2021. [Online]. Available: http://arxiv.org/abs/1406.5823.

[24] D. J. Barr, R. Levy, C. Scheepers, and H. J. Tily, 'Random effects structure for confirmatory hypothesis testing: Keep it maximal', $J$. Mem. Lang., vol. 68, no. 3, pp. 255-278, Apr. 2013, doi: 10.1016/j.jml.2012.11.001.

[25] R. H. B. Christensen, ordinal: Regression Models for Ordinal Data. 2019.

[26] R. V. Lenth, P. Buerkner, M. Herve, J. Love, H. Riebl, and H. Singmann, emmeans: Estimated Marginal Means, aka Least-Squares Means. 2021.
[27] E. B. Sandoval, J. Brandstetter, M. Obaid, and C. Bartneck, 'Reciprocity in Human-Robot Interaction: A Quantitative Approach Through the Prisoner's Dilemma and the Ultimatum Game', 2016, doi: 10.1007/s12369-015-0323-x.

[28] J. Xu and A. Howard, 'The Impact of First Impressions on HumanRobot Trust During Problem-Solving Scenarios', in 2018 27th IEEE International Symposium on Robot and Human Interactive Communication (RO-MAN), Aug. 2018, pp. 435-441, doi: 10.1109/ROMAN.2018.8525669.

[29] M. Paetzel, G. Perugia, and G. Castellano, 'The Persistence of First Impressions: The Effect of Repeated Interactions on the Perception of a Social Robot', in Proceedings of the 2020 ACM/IEEE International Conference on Human-Robot Interaction, New York, NY, USA, Mar. 2020, pp. 73-82, doi: 10.1145/3319502.3374786.

[30] G. Huisman, J. Kolkmeier, and D. Heylen, 'With Us or Against Us: Simulated Social Touch by Virtual Agents in a Cooperative or Competitive Setting', in Intelligent Virtual Agents, Cham, 2014, pp. 204-213, doi: 10.1007/978-3-319-09767-1_25.

[31] M. L. Walters, K. L. Koay, D. S. Syrdal, K. Dautenhahn, and R. Te Boekhorst, 'Preferences and perceptions of robot appearance and embodiment in human-robot interaction trials', 2009, Accessed: Feb. 20, 2021. [Online]. Available: http://uhra.herts.ac.uk/handle/2299/9642.

[32] M. M. Scheunemann, R. H. Cuijpers, and C. Salge, 'Warmth and Competence to Predict Human Preference of Robot Behavior in Physical Human-Robot Interaction', in 2020 29th IEEE International Conference on Robot and Human Interactive Communication (ROMAN), Aug. 2020, pp. 1340-1347, doi: 10.1109/ROMAN47096.2020.9223478.

[33] B. Mutlu, S. Osman, J. Forlizzi, J. Hodgins, and S. Kiesler, 'Perceptions of ASIMO: an exploration on co-operation and competition with humans and humanoid robots', in Proceedings of the 1st ACM SIGCHI/SIGART conference on Human-robot interaction, New York, NY, USA, Mar. 2006, pp. 351-352, doi: 10.1145/1121241.1121311.

[34] T.-Y. Hsieh, B. Chaudhury, and E. S. Cross, 'Human-robot cooperation in economic games: People show strong reciprocity but conditional prosociality toward robots'. PsyArXiv, Jul. 08, 2020, doi: 10.31234/osf.io/q6pv7.

[35] R. Hortensius and E. S. Cross, 'From automata to animate beings: the scope and limits of attributing socialness to artificial agents', Ann. N. Y. Acad. Sci., vol. 1426, no. 1, Art. no. 1, Aug. 2018, doi: 10.1111/nyas. 13727.

[36] D. Bridges, A. Pitiot, M. R. MacAskill, and J. W. Peirce, 'The timing mega-study: comparing a range of experiment generators, both labbased and online', PeerJ, vol. 8, p. e9414, Jul. 2020, doi: 10.7717/peerj.9414.

[37] J. R. de Leeuw and B. A. Motz, 'Psychophysics in a Web browser? Comparing response times collected with JavaScript and Psychophysics Toolbox in a visual search task', Behav. Res. Methods, vol. 48, no. 1, pp. 1-12, Mar. 2016, doi: 10.3758/s13428-015-0567-2.

[38] R. Miller, K. Schmidt, C. Kirschbaum, and S. Enge, 'Comparability, stability, and reliability of internet-based mental chronometry in domestic and laboratory settings', Behav. Res. Methods, vol. 50, no. 4, pp. 1345-1358, Aug. 2018, doi: 10.3758/s13428-018-1036-5.

[39] J. Wainer, D. J. Feil-seifer, D. A. Shell, and M. J. Mataric, 'The role of physical embodiment in human-robot interaction', in ROMAN 2006 - The 15th IEEE International Symposium on Robot and Human Interactive Communication, Sep. 2006, pp. 117-122, doi: 10.1109/ROMAN.2006.314404

[40] A. Wykowska, T. Chaminade, and G. Cheng, 'Embodied artificial agents for understanding human social cognition', Philos. Trans. $R$. Soc. B Biol. Sci., vol. 371, no. 1693, p. 20150375, May 2016, doi: 10.1098/rstb.2015.0375.

[41] A. Henschel, R. Hortensius, and E. S. Cross, 'Social Cognition in the Age of Human-Robot Interaction', Trends Neurosci., vol. 43, no. 6, pp. 373-384, Jun. 2020, doi: 10.1016/j.tins.2020.03.013. 